\title{
Should We Stop Using Gastric Residual Volumes?
}

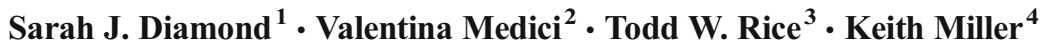

Published online: 10 July 2015

(C) Springer Science+Business Media New York 2015

\begin{abstract}
Early enteral nutrition support is an important component in the management of critically ill patients. However, gastrointestinal tolerance and gastric motility are frequently impaired in this population. Intolerance of enteral nutrition may present clinically as vomiting, aspiration, and abdominal distension. Concerns for the association between gastrointestinal intolerance and the development of ventilator-associated pneumonia have historically led to close monitoring of patients' tolerance of enteral feeding using various metrics. The measurement of gastric residual volumes (GRV) is the most commonly used surrogate marker for gastrointestinal intolerance in intensive care units. As the name implies, GRV is the volume of enteral formula remaining in a patient's stomach after cessation of enteral nutrition at various, predetermined time intervals. Despite the logical rationale, there is increasing evidence that monitoring gastric residual volumes may not improve patient outcomes in the critically ill. In this review, we will describe the components of normal gastric motor physiology and review abnormal physiology
\end{abstract}

This article is part of the Topical Collection on Gastroenterology and Nutrition

Sarah J. Diamond

diamonsa@ohsu.edu

1 Division of Gastroenterology and Hepatology, Oregon Health and Science University, 3181 SW Sam Jackson Blvd, L461, Portland, OR 97239, USA

2 Division of Gastroenterology and Hepatology, University of California Davis, Davis, CA, USA

3 Division of Allergy, Pulmonary, and Critical Care Medicine, Vanderbilt University, Nashville, TN, USA

4 Department of Surgery at University of Louisville, Louisville, KY, USA and dysfunction observed during critical illness. Finally, we will examine the evidence that both supports and refutes the routine use of gastric residual volumes and suggest specific recommendations about the future utilization of this practice.

Keywords Gastric residual volume · GRV · Enteral nutrition · Gastric dysmotility

\section{Introduction}

Early enteral nutrition is an important component in the management of critically ill patients. There remains fairly widespread and universal agreement among international nutrition guidelines that, if feasible, enteral nutrition should be provided within the first 1 to 2 days of admission to the intensive care unit $[1,2]$. Despite this consensus, the implementation of this standard practice may be complicated by clinical concerns of intolerance to gastric feedings [3]. Gastric dysmotility is common in critical illness but the oft-used measurements of gastric residual volume (GRV) to assess for gastric intolerance may be falling out of favor [4, 5]. Elevated GRV remains one of the most common reasons for holding enteral feedings and as such, one of the biggest obstacles to achieving goal enteral feeding rates $[6,7,8 \bullet \bullet]$.

\section{Physiology of Gastric Emptying}

To better understand the import and impairment of gastric motility in the critical care population, the normal physiology of gastric emptying must be understood. Normal gastric emptying depends on a complex, coordinated relationship between the gastric and small bowel musculature and the central nervous system. Various gut hormones play a crucial role in this 
normal physiology as well. The interstitial cells of Cajal (ICC), located in the greater curvature of the stomach, are "pacemaker cells," which stimulate electrical and mechanical activities in the smooth muscle cells of the stomach and are responsible for spontaneous motility [9].

The musculature of the stomach relaxes via reduction in muscle tension in response to distension. This process, known as accommodation, allows for increased capacity for food and liquid [10]. Peristalsis begins in the proximal stomach and occurs as two distinct types of contractions: slow, sustained contractions, and rapid, phasic contractions [11]. Slow waves originate in the pacemaker cells and are responsible for basal gastric tone. Peristaltic waves advance in increasing speed toward the antrum facilitating the physical churning and breakdown of food particles. This powerful antral contraction is mediated by the vagus nerve. Gastric emptying of liquids is mediated predominantly by change in amplitude of the slow, sustained contractions in the proximal stomach [11]. Gastric emptying of solids is largely driven by a decrease in the size of the gastric fundus and body, as the stomach slowly recovers wall tension that had decreased with the adaptive relaxation or accommodation in response to filling [10].

Multiple hormones are essential in the regulation of gut motility including cholecystokinin (CCK), peptide YY (PYY), motilin, glucagon-like peptide-1 (GLP-1), and ghrelin . Glucagon and incretins (amylin and GLP-1) slow gastric emptying to allow for slow food delivery to facilitate digestion and post-prandial glycemic control [12]. Ghrelin, on the other hand, has a promotility effect; levels increase in the plasma during fasting and decrease after ingestion of food $[13,14]$. Coordination of these multiple hormones is integral to the normal function and emptying of the stomach.

\section{Evaluation of Gastric Emptying}

There are multiple tests available to the clinician that can be used to evaluate gastric emptying. These tests are useful to quantify gastric emptying; some also assess motor function and myoelectrical activity of the stomach. Gastric emptying scintigraphy is considered the gold standard for the diagnosis of gastroparesis because this test quantifies the emptying of a solid meal [15]. In order to obtain accurate results, specific procedures need to be followed. Tests under $4 \mathrm{~h}$ in length may be unreliable due to wide variation in normal gastric emptying. Day-to-day variability in gastric emptying may also affect results. In addition to common gastric scintigraphy, various other indirect modalities are used to evaluate gastric emptying. These indirect tests include carbon-labeled breath tests, tests of drug absorption (paracetamol), and tests of carbohydrate malabsorption. Additional tests of gastric function include ultrasound, MRI, antroduodenal manometry, and gastric barostat [15]. In 2006, the FDA approved the wireless
motility/pH capsule for the assessment of regional and whole gut transit [16]. This wireless capsule uses changes in $\mathrm{pH}$ and temperature within the gastrointestinal lumen to assess gastric emptying time (GET), small bowel transit time (SBTT), colonic transit time (CTT), and whole gut transit time (WGTT). The capsule can also record intraluminal pressure and, therefore, provide pressure patterns in regions of the gastrointestinal tract [16].

While these tests perform accurately and precisely to measure gastric emptying in the ambulatory population, the assessment of gastric emptying in the critically ill population poses unique challenges and thereby may limit the utility of standard testing of gastric emptying in this population [17]. An appropriate and logistically applicable test to quantify gastric emptying in the critically ill patient remains elusive.

\section{Effects of Critical Illness on Gastric Emptying}

Critical illness has a profound effect on the normal physiology of gastric emptying. Dis-coordination and diminished functional association between proximal and distal parts of the stomach leads to altered gastric motility in this population [18].

The reasons for these alterations in normal physiology are multiple. One of the most common causes of altered gastric motility in the intensive care unit is medications. Various sedative medications have variable and deleterious effects on gastric emptying [19]. Some other common pharmacologic culprits that can delay gastric emptying include opioid analgesics, anti-cholinergics, tricyclic antidepressants, calciumchannel blockers, octreotide, proton pump inhibitors, H2receptor antagonists, fiber supplementation, beta-adrenergic receptor antagonists, and diphenhydramine among many others [15]. As is readily recognized from the above list, these medications are among those commonly administered in the setting of critical illness. There are many other reasons for altered gastric physiology in the critically ill population. Decreased blood flow from systemic hypoperfusion and the use of vasopressor medications that divert blood flow away from the intestine can also alter gastric and small bowel motility. In addition, surgery, systemic inflammatory response syndrome, and sepsis produce a cytokine response that may directly affect smooth muscle and the neuromuscular response in the GI tract leading to impaired motility [20].

Prior reports have cited wide variability in the proportion of critically ill and mechanically ventilated patients with delayed gastric emptying that range anywhere from 50 to $80 \%$ [21, $22 \bullet \bullet]$. While gastric emptying is inarguably delayed in periods of critical illness, the exact prevalence and severity may be poorly understood due to the difficulty in measuring gastric emptying in this specific population as previously discussed [17]. Standard measurement techniques are limited in the 
critical care setting. While gastric scintigraphy has been used to assess gastric emptying in the critically ill population, patient factors (mechanical ventilation, inability to transport, unpredictable clinical course) and facility factors (confined spaces, bulky equipment) limit its real-world utility [17]. Indirect testing may be more easily performed; however, variable protocols and imprecise pharmacokinetics affect the reliability of the results [17]. Gastric residual volume (GRV) is used as a default surrogate marker of gastric function and gastric emptying in the critical care setting because of these limitations with other direct and indirect measurements of gastric emptying.

\section{Limitations in the Use of Gastric Residual Volume}

As the name indicates, gastric residual volume is the volume of enteral formula remaining in a patient's stomach after cessation of enteral nutrition at various, predetermined time intervals. This volume is the volume of tube feed formula remaining in the stomach, measured by aspirating contents from the orogastric, nasogastric, or percutaneous gastrostomy tube using a syringe. While these general principles of measurement are fairly constant, the protocol and process used to perform this test are variable [3]. Furthermore, multiple factors affect the results [5]. The size of the syringe used to perform the test will influence the measurement; a larger bore syringe may be more likely to draw back a larger volume of gastric contents than a smaller syringe. The size and type of tubing used in the enteral feeding system has been demonstrated to affect the measurement of GRV [23, 24]. The size, placement, and material of the tubing as well as the number and placement of side ports will also affect the results [25]. Patientrelated factors contribute as well including the position of the patient, the administration of various common medications affecting motility and gastric secretions, and intra-abdominal pressure of the patient $[5,26]$. Given the variability of technique, the accuracy of this measurement is limited. Even in a highly controlled, experimental setting, using a mechanized device to aspirate a known volume of enteral formula from a cup led to a wide variance in the volume measured [27•].

There remains no consensus on the "normal" or safe GRV in the critically ill patient and importantly, the measurement of GRV is neither standardized nor validated [28]. Protocols vary widely among intensive care units and no clear "normal" or "expected" value has been widely agreed upon [1]. In a study that included 20 normal, healthy volunteers, at least $100 \mathrm{cc}$ of fluid remained in the stomach after aspiration in $40 \%$ of normal, healthy individuals compared to $50 \%$ of critically ill patients included in the study [26]. In a survey of 2298 critical care nurses, the most frequently cited GRV threshold value for interrupting feedings was $200 \mathrm{~mL}$ ( $36.5 \%$ of respondents reported interrupting feeds at this GRV). A quarter of respondents reported interrupting feeds at a GRV of $250 \mathrm{~mL}$, a quarter interrupted feeds at $150 \mathrm{~mL}$ or less, and only $12.6 \%$ of respondents interrupted feeds at a GRV of $500 \mathrm{~mL}$ [3]. Furthermore, while "acceptable" cutoff values vary widely, there is also no clarification on how to best use these cutoff values: does the GRV represent the value below which it is safe to advance feedings or a threshold value at which enteral feeding should be discontinued [1]? Practice also varies in replacing the volume that is removed in measuring the GRV. Some discard this volume, others return it to the patient, while others return part of it to a certain volume and discard the remainder. Similarly, practice is also inconsistent on how to handle GRV measurements in patients being fed via postpyloric feeding tubes. Some institutions continue to try to measure residual volumes (although not likely gastric) via the smaller bore post-pyloric tube. Others only measure GRV during post-pyloric feeding if the patient has a separate port or tube terminating in the stomach and others do not measure GRV during post-pyloric feedings.

This wide practice variability may stem from inconsistent messages regarding the clinical relevance of this tool. Despite the widespread reliance on this metric, there is variable association between high GRV and clinically relevant outcomes like aspiration or pneumonia [1]. Prior studies measuring yellow colorimetric microspheres in tracheal secretions have failed to show clear association between a wide range of values for GRV (from 0-50 mL up to $400-500 \mathrm{~mL}$ ) and aspiration events [29]. Two additional studies have sought an association between high gastric residual volumes and have found only a weak correlation [30, 31].

When considering the utility of GRV measurement, a common belief remains that high gastric residual volumes result in an increased incidence of pneumonia. Not only is there no convincing association between high GRV and aspiration events but there also have been no studies that have demonstrated that monitoring GRV actually reduces ventilatorassociated pneumonias (VAPs) [8••]. A systematic review of six randomized controlled trials examining the use of various threshold values of GRV to guide enteral nutrition found no association between the magnitude of GRV and the rate of complications such as frequency of vomiting, aspiration, and pneumonia (Table 1) [22••].

\section{Support Against the Use of Gastric Residual Volumes}

There have been several studies supporting the concept that routine checking of gastric residual volumes does not improve clinically important outcomes in the critical care setting. These studies have examined the consequences of significantly raising the proposed "cutoff values" in addition to abandoning the practice entirely. In an attempt to compare the effects of increasing the limit for GRV in the adequacy 
Table 1 Prospective randomized studies examining various threshold values for GRV to guide enteral nutrition

\begin{tabular}{|c|c|c|c|c|}
\hline $\begin{array}{l}\text { Author, year of } \\
\text { publication }\end{array}$ & $\begin{array}{l}\text { \# of patients } \\
\text { enrolled }\end{array}$ & Patient population & GRV threshold & Outcomes \\
\hline Pinilla et al. 2001 [32] & 96 & Mixed ICU (50 \% surgical) & $\begin{array}{l}>150 \text { vs. } \\
>250 \mathrm{~mL}+\text { prokinetic }\end{array}$ & No difference in frequency of vomiting \\
\hline McClave et al. 2005 [29] & 40 & $\begin{array}{l}\text { Mixed ICU (62.5\% } \\
\text { surgical) }\end{array}$ & $>200$ vs. $>400 \mathrm{~mL}$ & $\begin{array}{l}\text { No difference in frequency of } \\
\text { aspiration }\end{array}$ \\
\hline Desachy et al. 2008 [33] & 100 & Mixed ICU (32 \% surgical) & $\begin{array}{c}\text { Frequency of GRV } \\
\quad>300-400 \mathrm{~mL}\end{array}$ & No difference in frequency of vomiting \\
\hline Montejo et al. 2010 [25] & 329 & Medical ICU & $>200$ vs. $>500 \mathrm{~mL}$ & $\begin{array}{l}\text { No difference in frequency of } \\
\text { aspiration }\end{array}$ \\
\hline Reignier et al. $2013[8 \bullet \bullet]$ & 449 & $\begin{array}{l}\text { Predominantly medical } \\
\text { ICU ( } 93 \% \text { medical) }\end{array}$ & $\begin{array}{l}>250 \mathrm{~mL} \text { vs. no threshold ( } \\
\text { vomiting only) }\end{array}$ & $\begin{array}{l}\text { No difference in frequency } \\
\text { of VAP }\end{array}$ \\
\hline
\end{tabular}

$G R V$ gastric residual volume, $V A P$ ventilator-associated pneumonia, $E D E N$ early vs. delayed enteral nutrition in ALI

of enteral nutrition, a randomized controlled trial was performed in 28 intensive care units in Spain [25]. Of 322 patients studied, 165 patients were randomized to a control group in which a threshold GRV of $200 \mathrm{~mL}$ was used to guide management and 157 patients were randomized to the intervention group in which a GRV of $500 \mathrm{~mL}$ was permitted. Patients managed with higher GRV thresholds received a higher percentage of prescribed enteral nutrition and reached their enteral nutrition goals faster than the control group. While the overall gastrointestinal complication rate was higher in the control group (63.6\%) compared to the intervention group $(47.8 \%)(p=0.004)$, this difference was largely driven by a higher rate of high gastric residual volumes in the control group. This remains a circular argument. There was no difference in vomiting between groups $(p=0.31)$ and perhaps most importantly, there was no difference in ICU-acquired pneumonia between groups ( 27.4 vs. $28.0 \%, p=0.88)$

A single-center observational, before and after study performed in a 15-bed intensive care unit in France, represents another study investigating the effects of measuring GRV on clinical outcomes [34•]. A total of 205 critically ill, ventilated patients were studied. The control group included 102 patients in which GRV was measured every $6 \mathrm{~h}$. The intervention group included 103 patients in which no gastric residual volumes were measured. The primary outcome of interest was ventilator-associated pneumonia (VAP), which was suspected based on predefined clinical criteria and was confirmed with positive sputum culture obtained through broncheoalveolar lavage. Of the 102 patients randomized to GRV measurements, 20 (19.6\%) developed VAP compared to 19 of the $103(18.4 \%)$ randomized to no measurement of GRV. There was no difference in the rates of development of VAP ( $p=$ 0.86 ) or in the rates of vomiting between these two groups $(p=0.87)$. The intervention group received a higher volume of enteral nutrition overall $(p=0.002)$ demonstrating that patients administered enteral nutrition without GRV monitoring receive more enteral nutrition without experiencing increased rates of vomiting or VAP.

Subsequently, these authors performed a large, multicenter non-inferiority study in nine critical care units in France to support the findings of their observational study [ $8 \bullet \bullet$. They randomized 452 ventilated patients to either the control group (in which GRV was measured every $6 \mathrm{~h}$ with a $50 \mathrm{cc}$ syringe) or the intervention group (in which no GRVs were measured). The primary outcome of interest was ventilator-associated pneumonia (VAP), which was suspected based on clinical features and radiologic evidence and confirmed with positive cultures from broncheoalveolar lavage. Of 222 patients randomized to the control group, 35 (15.8\%) developed VAP compared to 38 out of $227(16.7 \%)$ in the intervention group. Analysis of secondary outcomes did note a higher vomiting rate in patients cared for without residual gastric volume monitoring, albeit confounded by a lower use of prokinetic drugs. Enteral feeding delivery was better in the intervention group with a higher proportion of patients achieving caloric targets when GRVs were not measured. There was no difference in important clinical outcomes, such as ICU and hospital length of stay, organ failure scores, or mortality rates between the two groups. These data support that not measuring gastric residual volumes is non-inferior to the practice of frequent measurement and that eliminating residual gastric volume monitoring from standard care may be beneficial.

\section{Challenges of Implementing Change}

There is mounting evidence that the routine use of GRV in the critical care setting is of little clinical benefit [4]. Furthermore, while the practice is neither standardized nor validated, the measurement of GRV in intensive care units is exceedingly common. Of 2298 critical care nurses surveyed, $97.1 \%$ cited measurement of GRV as the most common monitoring for 
gastrointestinal tolerance in their units [3]. However, several barriers must be overcome to move away from this common practice. Current guidelines on the use and monitoring of enteral nutrition still support routine measuring of GRV in critically ill patients [1]. Measurement of GRVs is still widely taught in nursing school, resulting in most nurses thinking it has clinical usefulness in protecting patients. Unfortunately, this significant gap among evidence, practice, and teaching will need to be overcome with revision of practice guidelines, rewriting of ICU protocols, and willingness of practitioners and educators to change long-standing practice patterns.

\section{Conclusions}

In summary, evidence continues to mount that routine monitoring of gastric residual volumes in critically ill patients receiving enteral nutrition leads to decreased delivery of enteral nutrition without a correlating decrease in adverse events (namely ventilator-associated or hospital-acquired pneumonia or lengths of stay). Given the universally understood benefits of enteral nutrition in this population and the interference of monitoring GRV with this important practice, we recommend that the practice of routinely monitoring GRV be systematically stopped and anticipate that subsequent nutrition guidelines will support this important clinical change.

\section{Compliance with Ethics Guidelines}

Conflict of Interest Sarah J. Diamond declares that she has no conflict of interest.

Valentina Medici declares that she has no conflict of interest.

Todd W. Rice declares that he has no conflict of interest.

Keith Miller has received support through a speaker/faculty fellowship from the Nestlé Nutrition Institute.

Human and Animal Rights and Informed Consent This article does not contain any studies with human or animal subjects performed by any of the authors.

\section{References}

Papers of particular interest, published recently, have been highlighted as:

- Of importance

•- Of major importance

1. McClave SA, Martindale RG, Vanek VW, et al. Guidelines for the provision and assessment of nutrition support therapy in the adult critically ill patient: Society of Critical Care Medicine (SCCM) and American Society for Parenteral and Enteral Nutrition (A.S.P.E.N.). JPEN J Parenter Enteral Nutr. 2009;33(3):277-316.

2. Heyland DK, Dhaliwal R, Drover JW, Gramlich L, Dodek P, Canadian Critical Care Clinical Practice Guidelines Committee. Canadian clinical practice guidelines for nutrition support in mechanically ventilated, critically ill adult patients. JPEN J Parenter Enteral Nutr. 2003;27(5):355-73.

3. Metheny NA, Mills AC, Stewart BJ. Monitoring for intolerance to gastric tube feedings: a national survey. Am J Crit Care. 2012;21(2): e33-40.

4. Rice TW. Gastric residual volume: end of an era. JAMA. 2013;309(3):283-4.

5. Elke G, Felbinger TW, Heyland DK. Gastric residual volume in critically ill patients: a dead marker or still alive? Nutr Clin Pract. 2015;30(1):59-71.

6. National Heart, Lung, and Blood Institute Acute Respiratory Distress Syndrome (ARDS) Clinical Trials Network, Rice TW, Wheeler AP, et al. Initial trophic vs full enteral feeding in patients with acute lung injury: the EDEN randomized trial. JAMA. 2012;307(8):795-803.

7. Rice TW, Mogan S, Hays MA, Bernard GR, Jensen GL, Wheeler AP. Randomized trial of initial trophic versus full-energy enteral nutrition in mechanically ventilated patients with acute respiratory failure. Crit Care Med. 2011;39(5):967-74.

8.• Reignier J, Mercier E, Le Gouge A, et al. Effect of not monitoring residual gastric volume on risk of ventilator-associated pneumonia in adults receiving mechanical ventilation and early enteral feeding: a randomized controlled trial. JAMA. 2013;309(3):249-56. This randomized controlled trial shows that by not monitoring gastric residual volumes, patients are more likely to receive $100 \%$ of their calorie goal without an increase the risk of ventilator associated pneumonia, ICU stay length or mortality rate.

9. Takaki M. Gut pacemaker cells: the interstitial cells of Cajal (ICC). J Smooth Muscle Res. 2003;39(5):137-61.

10. Schulze KS. The imaging and modelling of the physical processes involved in digestion and absorption. Acta Physiol (Oxf). 2015;213(2):394-405.

11. Minami H, McCallum RW. The physiology and pathophysiology of gastric emptying in humans. Gastroenterology. 1984;86(6):1592610.

12. Camilleri M. Clinical practice. Diabetic gastroparesis. N Engl J Med. 2007;356(8):820-9.

13. Tack J, Depoortere I, Bisschops R, Verbeke K, Janssens J, Peeters T. Influence of ghrelin on gastric emptying and meal-related symptoms in idiopathic gastroparesis. Aliment Pharmacol Ther. 2005;22(9):847-53.

14. Murray CD, Martin NM, Patterson M, et al. Ghrelin enhances gastric emptying in diabetic gastroparesis: a double blind, placebo controlled, crossover study. Gut. 2005;54(12): 1693-8.

15. Parkman HP, Hasler WL, Fisher RS, American Gastroenterological Association. American gastroenterological association technical review on the diagnosis and treatment of gastroparesis. Gastroenterology. 2004;127(5):1592-622.

16. Saad RJ, Hasler WL. A technical review and clinical assessment of the wireless motility capsule. Gastroenterol Hepatol (N Y). 2011;7(12):795-804.

17. Kar P, Jones KL, Horowitz M, Chapman MJ, Deane AM. Measurement of gastric emptying in the critically ill. Clin Nutr. 2014;34(4):557-64.

18. Nguyen NQ, Fraser RJ, Bryant LK, Chapman M, Holloway RH Diminished functional association between proximal and distal gastric motility in critically ill patients. Intensive Care Med. 2008;34(7):1246-55.

19. Nguyen NQ, Chapman MJ, Fraser RJ, et al. The effects of sedation on gastric emptying and intra-gastric meal distribution in critical illness. Intensive Care Med. 2008;34(3):454-60.

20. Overhaus M, Togel S, Pezzone MA, Bauer AJ. Mechanisms of polymicrobial sepsis-induced ileus. Am J Physiol Gastrointest Liver Physiol. 2004;287(3):G685-94. 
21. Heyland DK, Tougas G, King D, Cook DJ. Impaired gastric emptying in mechanically ventilated, critically ill patients. Intensive Care Med. 1996;22(12):1339-44.

22.• Kuppinger DD, Rittler P, Hartl WH, Ruttinger D. Use of gastric residual volume to guide enteral nutrition in critically ill patients: a brief systematic review of clinical studies. Nutrition. 2013;29(9): 1075-9. This systematic review includes 6 randomized controlled trials and 6 prospective observational studies and demonstrates that for mechanically ventilated patients in a medical intensive care unit, monitoring gastric residual volumes is unnecessary to guide nutrition.

23. Metheny N, Reed L, Worseck M, Clark J. How to aspirate fluid from small-bore feeding tubes. Am J Nurs. 1993;93(5):86-8.

24. Metheny NA, Stewart J, Nuetzel G, Oliver D, Clouse RE. Effect of feeding-tube properties on residual volume measurements in tubefed patients. JPEN J Parenter Enteral Nutr. 2005;29(3):192-7.

25. Montejo JC, Minambres E, Bordeje L, et al. Gastric residual volume during enteral nutrition in ICU patients: the REGANE study. Intensive Care Med. 2010;36(8):1386-93.

26. McClave SA, Snider HL, Lowen CC, et al. Use of residual volume as a marker for enteral feeding intolerance: prospective blinded comparison with physical examination and radiographic findings. JPEN J Parenter Enteral Nutr. 1992;16(2):99-105.

27. Bartlett Ellis RJ, Fuehne J. Examination of accuracy in the assessment of gastric residual volume: a simulated, controlled study. JPEN J Parenter Enteral Nutr. 2015;39(4):434-40. This study demonstrates that even in controlled, experimental conditions, the process of aspiration performed to measure gastric residual volumes is inaccurate and imprecise.
28. Zaloga GP. The myth of the gastric residual volume. Crit Care Med. 2005;33(2):449-50.

29. McClave SA, Lukan JK, Stefater JA, et al. Poor validity of residual volumes as a marker for risk of aspiration in critically ill patients. Crit Care Med. 2005;33(2):324-30.

30. Metheny NA, Clouse RE, Chang YH, Stewart BJ, Oliver DA, Kollef MH. Tracheobronchial aspiration of gastric contents in critically ill tube-fed patients: frequency, outcomes, and risk factors. Crit Care Med. 2006;34(4):1007-15.

31. Metheny NA, Schallom L, Oliver DA, Clouse RE. Gastric residual volume and aspiration in critically ill patients receiving gastric feedings. Am J Crit Care. 2008;17(6):512-9. quiz 520.

32. Pinilla JC, Samphire J, Arnold C, Liu L, Thiessen B. Comparison of gastrointestinal tolerance to two enteral feeding protocols in critically ill patients: a prospective, randomized controlled trial. JPEN J Parenter Enteral Nutr. 2001;25(2):81-6.

33. Desachy A, Clavel M, Vuagnat A, Normand S, Gissot V, Francois B. Initial efficacy and tolerability of early enteral nutrition with immediate or gradual introduction in intubated patients. Intensive Care Med. 2008;34(6):1054-9.

34. Poulard F, Dimet J, Martin-Lefevre L, et al. Impact of not measuring residual gastric volume in mechanically ventilated patients receiving early enteral feeding: a prospective before-after study. JPEN J Parenter Enteral Nutr. 2010;34(2):125-30. This before and after study highlights the increased delivery of enteral nutrition in mechanically ventilated patients in whom gastric residual volume is not measured. 